\title{
Machine Learning Algorithm for Early Detection of Heart Diseases Using 3-Tier IoT Architecture
}

\author{
Y. Madan Reddy, B. Pavani
}

\begin{abstract}
Among the applications empowered by the Internet of Things (IoT), regular health monitoring framework is an important one. Wearable sensor gadgets utilized in IoT health monitoring framework have been producing huge amount of data on regular basis. The speed of data generation by IoT sensor gadgets is very high. Henceforth, the volume of data generated from the IoT-based health monitoring framework is also very high. So as to overcome this problem, this paper proposes adaptable three-tier architecture to store and process such immense volume of wearable sensor data. Tier 1 focuses on gathering of data from IoT wearable sensor gadgets. Tier 2 employs Apache HBase for storing substantial volume of wearable IoT sensor data in cloud computing. Likewise, Tier-3 utilizes Apache Mahout for building up logistic regression-based prediction model for heart related issues. At long last, ROC examination is performed to identify the most significant clinical parameters to get heart diseases.
\end{abstract}

\section{INTRODUCTION}

In recent past, there has been a noticeable increase in the quantity of wearable gadgets for checking the patients' health and fitness activities on a regular basis[1]. This has a long haul impact on the recording of health and clinical support of patient's physiological information. This progression additionally helps the provision of more details identifying with the daily routine and physical examination. Amid the health monitoring period, IoT wearable gadgets are appended with the human body to track the different health measurements that incorporate blood pressure, heart rate, body temperature, respiratory rate, blood circulation level, body pain and blood glucose level [2]. The data gathered from the IoT-based wearable gadgets are stored in a clinical database for essential activity when the patients' health condition weakens.

Generally, conventional structured query language based databases are utilized in IoT health monitoring system to store clinical data. There has been an expansion in the variety and quantity of IoT-based health monitoring gadgets lately. Thus, the conventional data processing techniques and tools are not being utilized to store sensor data of high volume created by different IoT gadgets [3]. Scalable NOSQL (non structured query language ) databases must be utilized in the IoT-based health monitoring system. Analysts have begun the utilization of bigdata and NOSQL in different IoT applications. In this application, the proposed health

Revised Manuscript Received on 14 August, 2019.

Y.Madan Reddy, Assistant Professor, Department of CSE,MallaReddy Engineering College for Women,Hyderabad, Telangana, India, Emails: madanreddyyen@gmail.com,

B.Pavani, Assistant Professor,Department of CSE, MallaReddy College of Engineering \& Technology, Hyderabad, Telangana,India, Emails: pavanireddy523@gmail.com checking framework consistently watches the person's health condition. At the point when, the health measurements, for example, ECG, respiratory rate, pulse, perspiring, body temperature, blood pressure and heart sound go past standard values, the IoT gadgets send an ready message with the observed health measures to the doctor and other important people.

Sun et al. have built up the IoT-based tailings dam checking framework to monitor emergency situations in a tailings dam [5]. In this methodology the cloud computing based adaptable methodology is used for taking the necessary action when emergency situations arise.

Rohokale et al. have developed IoT-based health monitoring system to watch health parameters, for example, hemoglobin (HB), blood pressure (BP), glucose and irregular cell development [6]. The current methodologies utilize traditions databases and tools to process the immense volume of sensor data generated from IoT gadgets. Subsequently, there is a need to build up an effective and versatile design that stores and in addition breaks down the tremendous volume of clinical data. This paper proposes a adaptable big data based IoT health checking framework for tending to this issue.

The proposed IoT-based structure is interconnected with cloud computing technology to build scalability and accessibility.

Further, the proposed design utilizes Apache HBase to store the immense volume of the sensor data in the cloud. The person's health data is gathered with the help of RFID and 5G mobile networks. In addition, Apache Mahout is utilized in the proposed health monitoring system for building the calculated relapse based forecast show for heart diseases. Finally, the performance of the forecast demonstrate is nearly dissected with the assistance of different performance assessment measurements. The figured outcomes, for example, throughput, affectability, precision and f-measure are utilized for exhibiting the productivity and execution of the proposed IoT-based nonstop health monitoring system.

The proposed IoT-based nonstop health monitoring system is illustrated as follows: Section 1 depicts the introduction to IoT-based health monitoring system. Section 2 audits the ongoing works done in IoT-based healthcare systems. The proposed IoT-based ceaseless health monitoring system is illustrated in Section 3. Result and performance assessment are depicted in Sections 4 and 5 separately. Section 6 concludes the paper. 


\section{Machine Learning Algorithm for Early Detection of Heart Diseases Using 3-Tier IoT Architecture}

\section{RELATED WORK}

The Internet of Things (IoT) is an interconnection of different physical objects for watching the physical events on a regular basis. The connected IoT devices speak with one another with the assistance of cutting edge wireless networks and sensors [7] . IPv4 Internet was utilized in the last decade to exchange data at high speed.

Headways in system network have helped upgrade of IPv4 Internet to IPv6 Internet with diminished postponement and reaction time. IoT-based systems pursue the layered engineering for exchange of the flag and correspondence between the gadgets. The layers that assume a critical job in system availability incorporate Application Layer, Communication Layer, Security Layer, Embedded Layer, Hardware Layer, Integration Layer and DB Layer. RFID labels, sensors and actuators are utilized broadly in IoT-based structures. Exceptional tending to plans are utilized in IoT innovation for shared connection between IoT gadgets [8] .

The utilization of IoT technology in different fields has been on the expansion. For instance, Bäumer et al. have talked about the potential openings in utilizing Internet of Things in a business organization [9]. CodeBlue venture is the healthcare venture created by Harvard University. The basic job of the CodeBlue venture is in the observing of the people's health parameters, for example, ECG, EKG, EMG, SpO2, beat oximeter and Mica2 bits. Different electronic gadgets, for example, PDAs, PCs are utilized in the CodeBlue venture for vital activity from specialists and care holders when the patient's health condition break down [10]. Distributed and membership design are utilized in the CodeBlue task to convey the health status of the patients in continuous manner [11-13] . Researchers from the University of Virginia have created the Alarm-Net structure to monitor the patient's health status continuously. The three-level system design is utilized in the Alarm-Net task to detect the physiological parameters. IoT gadgets, for example, ECG, accelerometer and $\mathrm{SpO} 2$ sensors are attached to the human body in the primary level stage. The second level spotlights on watching the ecological parameters, for example, warmness, dampness, movement and brightness [14] . Ecological sensors are connected to living things to watch ecological parameters. Level 3 design is utilized for giving the system availability between the portals. Level three stage utilizes the Internet protocol (IP)- based system to empower wireless communication between source and destination $[15,16]$.

The primary level of Alarm-net is utilized for detecting the physiological parameters from a patient and exchanging the clinical information from the single-jump to the second level stage. The second level spotlights on sending the clinical information from level two to the third level using the shortest-path-first routing protocol. This project is broadly utilized for anticipating the emergency conditions of the patients based on the earlier health records. Essentially, MobiCare is another medicinal services venture created by Chakravorty et al. [17]. The project finds broad use in the checking of patient's health over a wide-region. This undertaking watches the clinical proportions of the patients meticulously and sends the physiological qualities to the doctor and the care holder with the assistance of fog and cloud computing. The IoT wearable sensor gadgets utilized in the MobiCare venture incorporate SpO2, ECG and blood oxygen $[18,19]$.

The MobiCare project detects the people's physiological data productively and sends it to the doctor and the care holder through a cell phone and PDA. CDMA or GPRS/UMTS wireless technologies are used for exchange of the clinical information gathered from the sensors to the doctor. The project utilizes HTTP POST protocol for sending the physiological data between the source and the destination. Correspondingly, PAM venture created by Blum et al. help perception of the mental health conditions of the patients [20]. The fundamental objective of the PAM venture is to identify the bipolar disorder (BP) ahead of time. Infrastructure and architecture based technologies are usedin the PAM project for building up the PAM-I and PAM-A blocks separately. PAM-I based framework utilizes electronic devices like PDA, PDAs, wearable IoT gadgets. PAM utilizes environmental sensors in the IoT-based health checking system to watch the sound and dust pollution in the environment [21]. Bluetooth with $5 \mathrm{G}$ portable systems is utilized for interfacing the different IoT sensors in the health monitoring system[22,23].

The intermediate servers make connection observation of the clinical data and store them into the database for clinical data examination. The current IoT-based health monitoring system have not utilized big data technology to store and process such huge volume of clinical data. So as to satisfy this gap, the proposed system utilizes Apache HBase to store the huge volume of clinical data in distributed way [24]. When the data is put away into the HBase, machine learning calculations are utilized for preparing such huge volume of data. This paper empowers the utilization of Apache Mahout based machine learning algorithms to build up the prediction model. MapReduce based logistic regression is proposed to model the early stage of heart illness. A comparative examination of the performance of the proposed prediction model is made utilizing other existing machine learning approaches. The test results demonstrate the viability of the proposed model.

\section{PROPOSED FRAMEWORK FOR IOT-BASED HEALTH MONITORING SYSTEM}

The proposed IoT-based Health Monitoring System comprises of a three-tier architecture to store and process enormous volume of wearable sensor data. Tier 1 focuses on gathering data from IoT wearable sensor gadgets. Tier 2 utilizes Apache HBase to store the huge volume of wearable IoT sensor data in the cloud. Furthermore, Tier-3 utilizes the Apache Mahout to build up the logistic regression-based prediction model for heart diseases. Fig. 1 shows the proposed framework for IoT-based Health Monitoring System. Fig. 2 shows the work process for the proposed framework. 


\section{a.TIER 1: DATA GATHERING}

The proposed IoT-based health monitoring system comprises of three blocks, specifically, datagatheringblock, data storage block and data analytics block. Datagatheringblock is used for gathering the people's physiological data using wearable IoT sensor gadgets. IoT wearable gadgets connected to the human body gather the patient's clinical data in a nonstop manner. At the point when the clinical proportion of the people surpasses its normal value, the gadgets send an alert with clinical value to the doctor and the care holder. The alert messages and clinical values are gathered and stored in database on regular basis. The proposed system utilizes $5 \mathrm{G}$ mobile networks for transfering the clinical data into the clinical database for empowering the necessary action in a crisis circumstance. Algorithm1 represents the IoT gadget initialization and continuous monitoring strategy and demonstrates the necessary stepsto move the clinical data( clinical_data.csv ) seen from IoT gadgets into the Amazon S3 container ( health data). The proposed IoT-based health monitoring system uses 's $3 \mathrm{cmd}$ ' strategy for exchange of the clinical data from the local disk into the cloud database.

\section{b.TIER 2: DATA STORAGE}

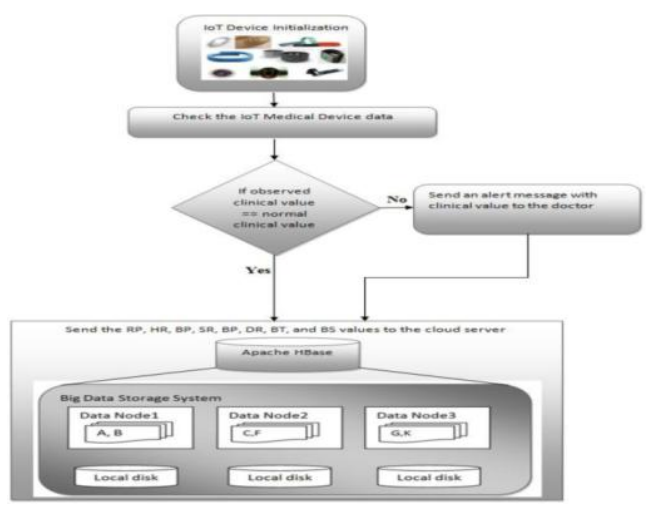

Normally, IoT gadgets have the target of sending clinical data constantly. It is hard to store and process such huge sum of data by traditional data processing tools and techniques. The proposed system uses big data technologies to store the clinical data in a distributed way. Apache HBase plays a critical job in storing data in distributed way. Physical devices and PCs are not adequate to store the huge volume of data produced from the IoT wearable gadgets. The proposed IoT-based health monitoring system takes care of the problems using cloud computing technologies for adaptability and elasticity. An account is created with Amazon to get the virtual machines with Apache HBase database accessibility. At first, clinical data gathered from the human body is transferred to the Amazon storage service S3 with the assistance of the 's3cmd utility' technique. Consequently, when the clinical measure of the people's surpasses its normal value, the IoT gadgets send the clinical measures to the Amazon S3. Contrasted with Amazon S3 storage, Apache HBase gives a versatile data storage system a dispersed way. Thus, the clinical data is transfered from the Amazon S3 to Apache HBase. The proposed health monitoring system uses Apache Pig to transfer the clinical data from Amazon S3 to Apache HBase.
Apache Pig is broadly used to extract, load and transform the huge semi-structured, unstructured and structured data. PigLatin is a language utilized in Apache Pig for data exchange. Algorithm 2 indicates the PigLatin program for data transmission of the clinical data between the Amazon S3 can and Apache HBase. Fig. 3 is a data flow graph for the proposed system. Wearable IoT gadgets fixed to the human body to gather the person's physiological data. These gadgets generate huge volume of data that can't be stored in the traditional databases. Subsequently, HDFS is used for storing huge volume of data in a versatile manner. Algorithm 3 is utilized for exchange of data between Amazon S3 and Apache HBase.

\section{c.TIER 3: DATA ANALYTICS}

Data analytics block is used for the developing of the prediction model using logistic regression. The proposed system uses Apache Mahout based machine learning libraries for implementing logistic regression, which is one of the machine learning strategies for predicting the coefficient between a dependent variable and one or more independent variables. logistic regression works in indistinguishable way as linear regression, with the difference of a independent variable. In linear regression, dependent variable Y's and independent variable X's are in the form of numbers, while, in logistic regression, the independent variable $\mathrm{X}$ 's may likewise be categorical values and the dependent variable Y's are coded as 1 in maximum cases (for the individuals who are present) 0 (for the individuals who are absent). Multiple logistic regression is defines by,

$\operatorname{logit}(p)=b 0+b 1 X 1+b 2 X 2+\ldots+b k X k$

Where,

$\mathrm{p}=$ denotes the probability of presence of the dependent variable (heart disease 0 or 1 )

$\mathrm{b}=$ denotes the coefficients,

$\mathrm{Xi}=$ denotes independent variables,

$\mathrm{i}=$ Number of clinical parameters
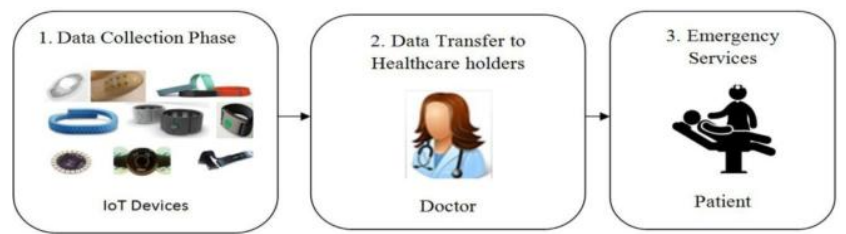

$\mathrm{X} 1$ = Respiratory Rate (RP),

$\mathrm{X} 2$ = Heart rate $(\mathrm{HR})$,

X3 = Blood Pressure-Systolic Range (BP-SR),

X4 = Blood Pressure - Diastolic Range (BP-DR),

X5 = Body Temperature (BT),

X6 = Blood Sugar - Fasting (BS-F),

X7 = Blood Sugar - Post Meal (BS-PM).

Logged odds are used for defining logistic transformation. The general formula for calculating of the odds ratio is defined by, 
A. p probability of presence of the var

Odds $=$

B. $(1-p)$ probability of absence of the var (2)

Whereas logged odds is defined by,

$$
\operatorname{logit}(p)=\ln \left(\begin{array}{l}
p \\
1-p
\end{array}\right.
$$

The conversion between the logged odds and probabilities are defined by,

$p=e b 0+b 1 X 1+b 2 X 2+\ldots+b k X k$

$1+e b 0+b 1 X 1+b 2 X 2+\ldots+b k X k$

The proposed IoT-based health monitoring framework uses logistic regression to develop the prediction model for monitoring heart diseases at the early stage. The Apache Mahout is used with Elastic MapReduce (EMR) framework for the development of the prediction model in cloud computing. Once the clinical data is stored in the Apache HBase, the Mahout- based logistic regression uses the prior clinical records for developing a prediction model. Pseudo code 1 represents the implementation steps for the Apache Mahout in the Hadoop Distributed File System (HDFS). The implementation is done in the Linux based distributed environment.

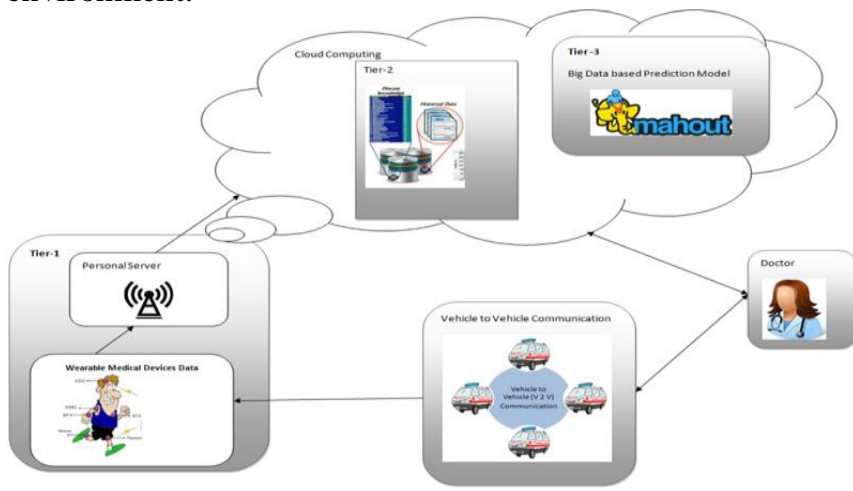

Fig 1. Proposed framework for IoT-based health monitoring system.

Fig. 3. Data flow diagram for the proposed framework.

Pseudo code 1: Mahout Prediction Model Development in the Linux Environment

Step1: Install Hadoop andJava

Step2: Install maven 3

sudo apt-get installmaven

Step3: mvn installationverification

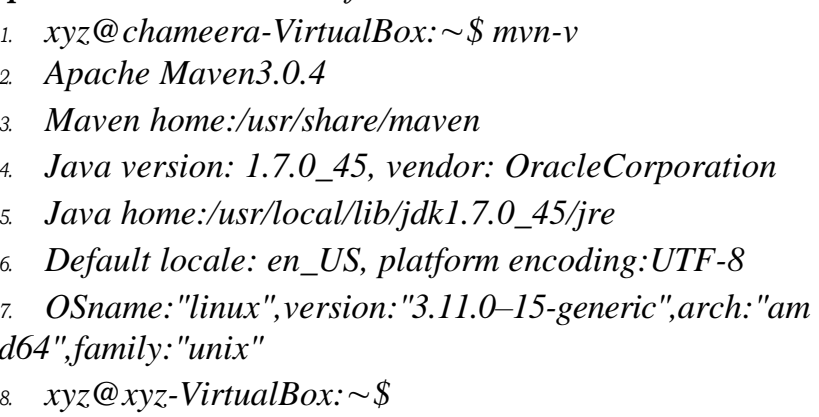

Step4: Install svn

1. Open terminal $(\mathrm{Ctrl}+\mathrm{Alt}+\mathrm{T})$

2.sudo apt-get installsubversion

Step5: Verify that the installation

1.xyz@xyz-VirtualBox: \$svn-version svn,version 1.7.9 (r1462340) compiled Oct 15 2013, 12:40:34

Copyright (C) 2013 The Apache Software Foundation.

Step6: Install Mahout

1. Select the directory to install Apache Mahout cd/home/xyz/home

2. Make new directory mkdirmahout

3. Find the new directory cdmahout

4. UseSubversiontocheckoutthecode

svn co http://svn.apache.orgftrepos/asf/mahout/trunk

5. Find the trunk directory.

cdtrunk

6. Build the Apache mahout

mvn install

mvn -DskipTests

Pseudo code 2 represents the steps to train the logistic regression in Linux based distributed environment. Pseudo code 3 represents the performance of the logistic regression-based prediction model to identify the heart disease.

\section{Pseudo code 2: Mahout Prediction Model Training Phase Step1: Find the Mahout Path}

cd /home/xyz/home

cd mahout

\section{Step2: Prediction Model Training}

mahout org.apache.mahou t.classifier.sgd. TrainLogistic -passes 1 -rate 1-lambda 0.5 -input donut.csv-features 21 -output donut.model - target color -categories 2 predictors $x$ y $x x$ xy yy a b c-types $n n$

\section{Pseudo code 3: Mahout Prediction Model \\ Prediction Phase}

\section{Step1: Find the Mahout Path}

cd /home/xyz/home

cd mahout

\section{Step2: Prediction Model Training}

mahout org.apache.mahout.classifier.sgd.RunLogistic -input donut.csv -model donut.model -auc -scores -confusion

\section{RESULT AND DISCUSSION}

Table 1 depicts the predicted coefficient values for the logistic regression-based prediction model. Respiratory Rate (RP), Heart rate (HR), Blood Pressure (BP)-Systolic Range (SR) and Blood Pressure (BP)-Diastolic Range (DR) are identified as sig- nificant variables for heart disease on the basis of p-values. Body Temperature (BT), Blood Sugar (BS)-Fasting and Blood Sugar (BS)-Post Meal are considered as not significant variables for heart disease based on the p-values. In this study, seven attributes are used for performing the experiment [25] . 


\section{PERFORMANCE EVALUATION}

Sensitivity, specificity, positive likelihood ratio (PLR), negative likelihood ratio (NLR), disease prevalence (DP), positive predicted value (PPV) and negative predicted value (NPV) are calculated for evaluating of the prediction model.

The validations metric are defined by,

$$
\text { True Negative (TN) }
$$

\section{Algorithm 2}

\section{Store the IoT wearable sensor devices data into Amazon} S3 data store.

\section{Step1:IdentifytheSystemNameintheAmazonS3 \\ 2. Step 2: Create the directory in the Amazon S3} Name_of_the_System \$s3cmd mb s3://health_data Bucket 's3:// health_data/'created

3. Step 3: Use put method to store the clinical data into the Amazon S3 directory Name_of_the_System \$ s3cmd put clinical_data.csv.s3://health_data

4. Step 4: Visualize the log file of the clinicaldata sample-syslog.log -> s3:// health_data/clinical_data.csv

\section{Algorithm 3 \\ Data transformation between the Amazon S3 and Apache HBase.}

1. Data:ClinicaldatacollectedfromIoTsensordeviceswhich isstoredinAmazonS3

2. Input: Amazon S3 health dataTable

3. Output: Pig Data Load Tablequery

4. Step-1:Hadoopdistcps3n:health_data/clinical_data.c sv/user/patient1

5. Step-2: if (Table_Name /= NULL)then

6. Step-3:load '/user/patient/ clinical_data.csv' using PigStorage(',')as(add field name, field data type);

7. stored into hbase://clinical_data Table'usingorg.apache.pig.backend.hadoop.hbase.HBa seStorage

(add column family name patient ID: field column family data type Integer);

(addcolumnfamilynameRespiratoryRate(RP):fieldcolumnfa milydatatypeInteger);

(add column family name Heart rate (HR):field column family data typeInteger);

(add column family name Blood Pressure (BP):Systolic Range (SR):field column family data type Integer);

(add column family name Blood Pressure (BP):Diastolic Range (DR):field column family data type Integer);

(addcolumnfamilynameBodyTemperature(BT):fieldcolumnf amilydatatypeDouble);

(add column family name Blood Sugar (BS):field column family data type Integer);

(add column family name Blood Sugar (BS):Post Meal: field column family data type Integer);

8. Step-4: Return the Pig Data Load Table query

Fig. 4. True positive and false positive rate.

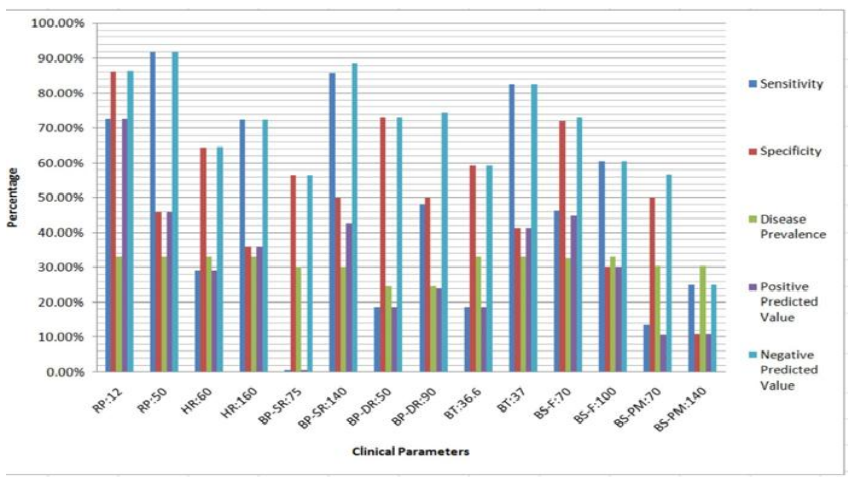

Fig 5:Performance evaluation metrics

\section{CONCLUSION}

This paper proposes a scalable IoT based three-tier architecture to process the sensor data and identify the most significant clinical parameters to get heart disease. The most significant clinical parameters that indicate impending heart disease are identified with the help of ROC analysis. Blood Sugar (BS)-Fasting and Blood Sugar (BS)-Post Meal are found to have positive correlation with heart disease. However, Respiratory Rate (RP), Heart rate (HR), Blood Pressure (BP): Systolic Range (SR), Blood Pressure (BP): Diastolic Range (DR) and Body Temperature (BT) are found to be negatively correlated with heart disease. The experimental results prove that Respiratory Rate (RP) at around 50 and 12 is highly significant in the indica- tion of the heart disease. Heart rate 160 is also indication of heart disease. Similarly, Blood Pressure (BP): Systolic Range and Body Temperature (BT) at 140 and 37 are also considered as a highly significant variable for heart problem indication. The future work of this study is to propose a continuous health monitoring system with a doctor on the move with an ambulance. An energy efficient node selection algorithm is identified for future work to choose the best mobile ambulance.

\section{REFERENCES}

1. Lorincz K, Malan DJ, Fulford-Jones TR, Nawoj A, Clavel A, Shnayder V, et al. Sensor networks for emergency response: challenges and opportunities.Pervasive Comput, IEEE2004;3(4):16-23.

2. Shafiq MZ, Ji L, Liu AX, Pang J, Wang J. A first look at cellular machine-to-machine traffic: large scale measurement and characterization. ACM SIG- METRICS Perform Eval Rev2012;40(1):65-76.

3. Verma PK, Verma R, Prakash A, Agrawal A, Naik K, Tripathi R, et al. Machine-to-Machine (M2M) communications: a survey. J Network Comput Appl2016;66:83-105.

4. Hassanalieragh M, Page A, Soyata T, Sharma G, Aktas M, Mateos G, et al. Health monitoring and management using internet-of-things (IoT) sensing with cloud-based processing: opportunities and challenges. In: Proceedings of 2015 IEEE international conference on services computing (SCC). IEEE;2015 June, p.285-292.

5. Sun E, Zhang X, Li Z. The Internet of Things (IOT) and cloud computing (CC) based tailings dam monitoring and pre-alarm system in mines. Saf Sci 2012;50(4):811-15.

6. Rohokale VM, Prasad NR, Prasad R. A cooperative Internet of Things (IoT) for rural healthcare monitoring and control. In: Wireless communication, vehicular technology, information theory and aerospace \& electronic systems technology (wireless VITAE), 2011 2nd international conference on. IEEE; February 2011. p.1-6.

\section{Published By:}

Blue Eyes Intelligence Engineering 
7. BaoyunW.ReviewonInternetofThings.JElectrMeasurInstrum2009; 23(12):1-7.

8. Doppler K, Rinne M, Wijting C, Ribeiro CB, Hugl K. Device-to-device communication as an underlay to LTE-advanced networks. IEEE Commun Mag 2009;47(12):42-9.

9. ManogaranG,LopezD.AGaussianprocessbasedbigdataprocess ingframeworkinclustercomputingenvironment.ClusterComput 2017:1-16.

10. Ishaq I, Carels D, Teklemariam GK, Hoebeke J, Abeele FVD, Poorter ED, et al. IETF standardization in the field of the Internet of Things (IoT): a survey. Networks2013;2(2):235-87.

11. KumarP,LeeHJ.Securityissuesinhealthcareapplicationsusi ngwirelessmedicalsensornetworks:asurvey.Sensors2011;1 2(1):55-91.

12. Manogaran G, Thota C, Kumar MV. MetaCloudDataStorage architecture for Big Data security in cloud computing. Procedia Comput Sci 2016Dec 31;87:128-33.

13. Manogaran G, Thota C, Lopez D, Vijayakumar V, Abbas KM, Sundarsekar R. Big data knowledge system in healthcare. In: Internet of Things and big datatechnologiesfornextgenerationhealthcare;2017.p.133.

14. Gubbi J, Buyya R, Marusic S, Palaniswami M. Internet of Things (IoT): a vision, architectural elements, and future directions. Future Gener Comput Syst 2013;29(7):1645-60.

15. WhitmoreA,AgarwalA,DaXuL.TheInternetofThings - asurveyofto picsandtrends.InfSystFront2015;17(2):261-74.

16. Manogaran G, Thota C, Lopez D, Vijayakumar V, Abbas KM, Sundarsekar R. Big data knowledge system in healthcare. In: Internet of Things and big datatechnologiesfornextgenerationhealthcare.SpringerInternati onalPublishing;2017.p.133-57.

17. VaratharajanR,ManogaranG,PriyanMK,BalasVE,BarnaC.Visuala nalysisofgeospatialhabitatsuitabilitymodelbasedoninversedis tanceweighting withpairedcomparisonanalysis.MultimedToolsAppl2017:1-21.

18. AtzoriL,IeraA,MorabitoG.TheInternetofThings:asurvey.ComputN etworks2010;54(15):2787-805.

19. Manogaran G, Thota C, Lopez D, Vijayakumar V, Abbas KM, Sundarsekar R. Big data knowledge system in healthcare. In: Internet of things and big datatechnologiesfornextgenerationhealthcare.SpringerInternati onalPublishing;2017.p.133-57.

20. Blum JM, Magill EH. The design and evaluation of personalised ambient mental health monitors. In: Proceedings of 7 th consumer communications and networking conference (CCNC). IEEE; 2010. p.1-5.

21. Bonomi F, Milito R, Zhu J, Addepalli S. Fog computing and its role in the internet of things. In: Proceedings of the first edition of the MCC workshop onMobilecloudcomputingACM,August;2012.p.13-16.

22. Manogaran G, Lopez D. Spatial cumulative sum algorithm with big data analytics for climate change detection. Comput Electr Eng 2017. http://dx.doi. org/10.1016/j.compeleceng.2017.04.006.

23. Priyan MK, Devi GU. Energy efficient node selection algorithm based on node performance index and random waypoint mobility model in internet of vehicles. Cluster Comput 2017:1-15.

24. Varatharajan R, Manogaran G, Priyan MK, Sundarasekar R. Wearable sensor devices for early detection of Alzheimer disease using dynamic time warping algorithm. Cluster Comput2017:1-10.

25. UCIMachineLearningRepository:HeartDiseaseDataSetArchiv e.ics.uci.edu.Retrieved22July2017,fromhttp://archive.ics.uci.e $\mathrm{du} / \mathrm{ml} /$ datasets/heart+ Disease. 